\title{
CIÊNCIA'NATURA
}

\section{A simple formula for the evaluation of value and position of ground level concentration from a point source}

\author{
${ }^{1}$ Tiziano Tirabassi e ${ }^{2}$ Daniela Buske \\ ${ }^{1}$ Institute of Atmospheric Science and Climate (ISAC-CNR), Bologna, Itália \\ ${ }^{2}$ Universidade Federal de Pelotas, Pelotas, RS, Brasil
}

\begin{abstract}
After setting realistic scenarios of the wind and diffusivity parameterizations the Ground Level Concentration is worked out by an analytical solution of the advection-diffusion equation, then an explicit approximate expression is provided for it allowing a simple expression for the position and value of the maximum.
\end{abstract}

Keywords: Air pollution modelling. Analytical solutions. Maximum ground level concentration.

\section{Resumo}

Depois de estabelecer cenários realistas das parametrizações do vento e da difusividade, a concentração do nivel do solo é elaborada por uma solução analítica da equação de advecção-difusão e, em seguida, é fornecida uma expressão simples para a posição e o valor do máximo de concentração no nível do solo.

Palavras-chave: Modelagem da poluição do ar. Soluções analiticas. Concentração máxima no nível do solo. 


\section{Introduction}

Irreversible consequences of air pollution in the Atmospheric Boundary Layer (ABL) demand for increasing real time environmental monitoring and control as a routine instrument as well as for cases of environmental accidents or even catastrophes. In order to evaluate such scenarios one needs fast procedures, which yield immediate results as for instance the ground level concentration of pollutants, especially the maximum concentration and its position. Although numerical simulation approaches may still be too slow to provide a map of concentrations in real time, when immediate decisions are necessary. However, analytical solutions for theoretical models are independent of a specific situation by parameter estimation. The computational evaluation of numerical data of the concentration field or for a set of position is then an instant task. In this line the present work presents a derivation of compact phenomenological formula extracted from the analytical GILTT (Generalized Integral Laplace Transform Technique) (Moreira et.al, 2009; Tirabassi et al., 2008; Buske et al., 2007) approach which permits to determinate the ground level concentration in terms of physical parameters.

\section{Turbulent Parameterization}

We restrict our discussion to simple vertical profiles of wind and eddy diffusivity, nevertheless still reasonably realistic and only for unstable regime. The choice of the vertical profile for the wind $u(z)$ is set to be following a power law (PANOFSKY and DUTTON, 1988):

$$
\frac{u(z)}{u_{1}}=\left(\frac{z}{z_{1}}\right)^{\alpha}
$$

where $u_{1}$ is the mean wind velocity at the height $z_{1}$, while $\alpha$ is an exponent related to the turbulence intensity (Irwin, 1979). On the quantitative side, results will be provided setting $\alpha=0.1$, and the reference wind $u_{1}(0.01 h)=3 m s^{-1} ;$ these values are quite consistent with the whole range of unstable regimes pointed out by Pasquill and Smith (1984).

The vertical diffusivity parameterization is chosen according to Pleim and Chang (1992), which for an unstable ABL it is given as:

$$
k_{z}(z)=k w_{*} z\left(1-\frac{z}{h}\right)
$$

where $h$ is the height of the ABL, $k$ is the von Karman constant which is set to 0.4 , and $w_{*}$ is the convective scaling parameter related to the Monin-Obukhov length $L_{M O}$ and the mechanical friction parameter $u_{*}$ as:

$$
w_{*}=u_{*}\left(\frac{h}{L_{M O}}\right)^{1 / 3}
$$

For convective scenarios $L_{M O}$ is limited to values such that the relationship $h / L_{M O}<-10$ holds. Finally $u_{*}$ is determined as (PANOFSKY and DUTTON, 1988; ZANNETTI, 1990)

$$
u_{*}=u_{1} k\left(\ln \frac{z_{1}}{z_{0}}-\psi(\xi)\right)^{-1}
$$

where $z_{0}$ is the roughness $\left(10^{-5} h\right)$. For an unstable ABL $\psi$ defined as

$$
\psi(\xi) \cong \ln \left[\left(\frac{1+\xi^{2}}{2}\right)\left(\frac{1+\xi}{2}\right)^{2}\right]-2 \arctan \xi+\frac{\pi}{2}
$$

and

$$
\xi=\left(1-16 \frac{z_{1}}{L_{M O}}\right)^{\frac{1}{4}}
$$

The chosen profiles, described in (1)-(6), ensure simple functions and still rather realistic horizontal wind $u(z)$ and diffusivity $k_{z}(z)$ inside and both edges of the ABL. 


\section{Evaluation of Ground Level Concentration}

Ground Level Concentration (GLC) will be reported in terms of the dimensionless GLC as follows:

$$
C_{G L C}(x)=C(x, 0) \frac{<u>h}{Q}
$$

where $\mathrm{Q}$ is the emission source and $\langle u\rangle$ is the vertically averaged wind introduced in Eq. (1)

$$
<u>=\frac{1}{h} \int_{0}^{h} u(z) d z
$$

If we consider the definition of $u$ profile in Eq.(1) we have

$$
<u>=\frac{u_{1}}{\alpha+1}\left(h / z_{1}\right)^{\alpha}
$$

Equation (7) has been introduced to obtain the unitary limit independent of a specific parameter choice

$$
\lim _{x \rightarrow \infty} C_{G L C}(x)=1
$$

according to the theoretical expectation for the two-dimensional ADE solution.

The choice of a profile depending approximation maintains the advantage of simplicity and permits for a specific case to explore the functional behaviours of the main physical parameters that drive atmospheric diffusion. To this end we introduce empirical parameters which are determined by fit procedures to best reproduce the exact solution.

Based on these facts, and being in mind the Gaussian solution and the GLC obtained with power low profile of wind and eddy diffusivity, the dimensionless GLC defined in Eq. (7) can be approximated as follows:

$$
C_{G L C}(x)=\left[1+\left(\frac{\kappa h}{\lambda x}\right)^{c}\right]^{b} \exp \left[-\frac{\left(\pi h_{S}\right)^{1+2 b c}}{h(\lambda x)^{2 b c}}\right]
$$

Due to the negative values assumed by the Monin-Obukhov length, in the following it will be defined as the positive dimensionless parameter $L_{M O}=-L_{M O} / h$. Parameters $b, c, \kappa$ and $\lambda$ have been determined by least squares fittings procedures on Eq. (10) against the analytical solution and these are:

$$
\begin{gathered}
b=h_{S}^{0 / \delta^{2}}+.17 \\
c=-5.48 h_{S}^{0 / 87}+4.73 \\
\kappa=\left(\frac{\alpha+1}{0.4277}\right)^{2.62} h_{S}^{0 / 61} \\
\lambda=\left(0.35 u_{1}\right)^{-1}(\alpha+1)^{-1.3} w_{*} h / .67
\end{gathered}
$$

where the variables with $\%$ normalized with respect to the ABL height $h\left(\right.$ e.g. $\left.h_{s}=h_{s} / h\right)$.

Equations (11) - (14) give the explicit dependency on the source height $h_{S}$, the wind parameters $\alpha$ (it compares in $k$ and $\lambda$ ), $u_{1}$ and the convection scaling parameter $w_{*}$ (it compares in $\lambda$, see Eq.(14)) which is related to the Monin-Obukhov length $L_{\|}$ and the friction parameter $u_{*}$ by the relationship (3).

From the explicit approximation for $C_{G L C}(x)$ one may evaluate the position where the maximum for GLC occurs, in fact putting equal to 0 the derivative of Eq.(10) in respect to $x$ and with the assumption that:

$$
\left(\frac{\kappa h}{\lambda x}\right)^{c}>>1
$$


we have:

$$
x_{M}^{2 b c}=2 \frac{\left(\pi h_{S}\right)^{1+2 b c}}{(h \lambda)^{2 b c}}
$$

Finally, putting $x_{M}$ in Eq.10, the corresponding Maximum Ground Level Concentration $\left(C_{M G L C}\left(x_{M}\right)\right)$ is:

$$
C_{M G L C}\left(x_{M}\right)=\left[1+\left(\frac{2 \kappa}{\left(2 \pi h_{S}^{\circ}\right)^{1+\frac{1}{2 b c}}}\right)^{c}\right]^{b} e^{-\frac{1}{2}}
$$

\section{Results}

In Figure $1 \mathrm{a}$ and $1 \mathrm{~b}$, the GLC versus $\not / 1$ is shown for $h_{S}^{0}=0.01,0.05,0.1$ (a-c), and $h_{S}^{0}=0.25,0.4,0.5$ (d-f). For each source height two extreme Monin-Obukhov lengths are set, corresponding to $L_{M O}=0.001,0.099$ (empty squares and triangles respectively). The GILTT-based GLC are superimposed with the approximation of Eq. (10) (dotted lines). Plots highlight that for near surface sources there is a slight mismatch between points and lines near the source position, where the horizontal gradient is most pronounced, logarithmic scales enhance such a discrepancy. As source height increases a higher matching results, including a fair reproduction of the position where the maximum GLC occurs. As the emitting source height $h_{S}$ increases the approximated function slightly underestimate the GILTT-based maximum. Such a discrepancy reflects the fact that condition (15) is no longer satisfied. No-

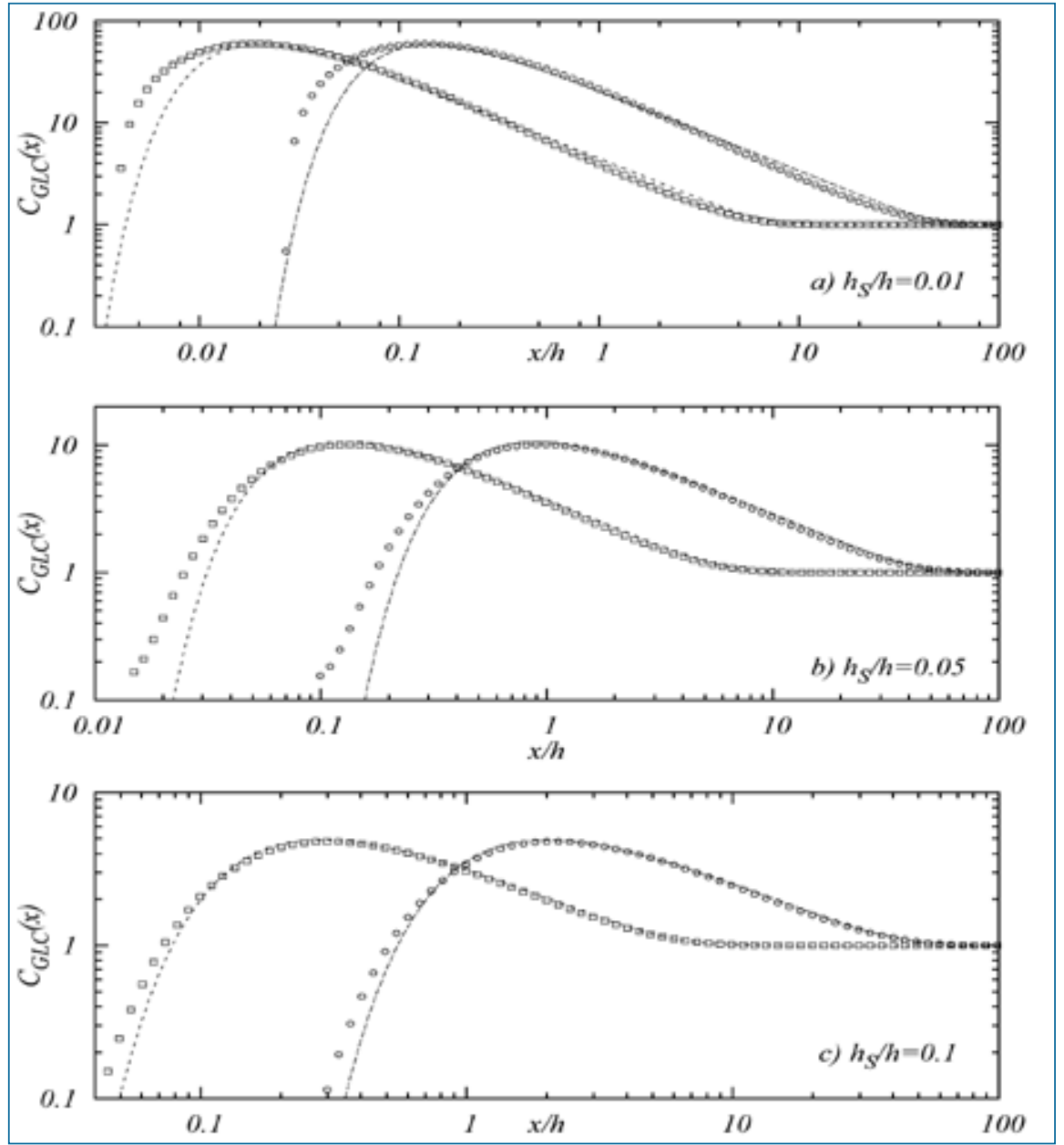

Figure 1a - The GLC is plot versus $2 /$ for several source heights. 


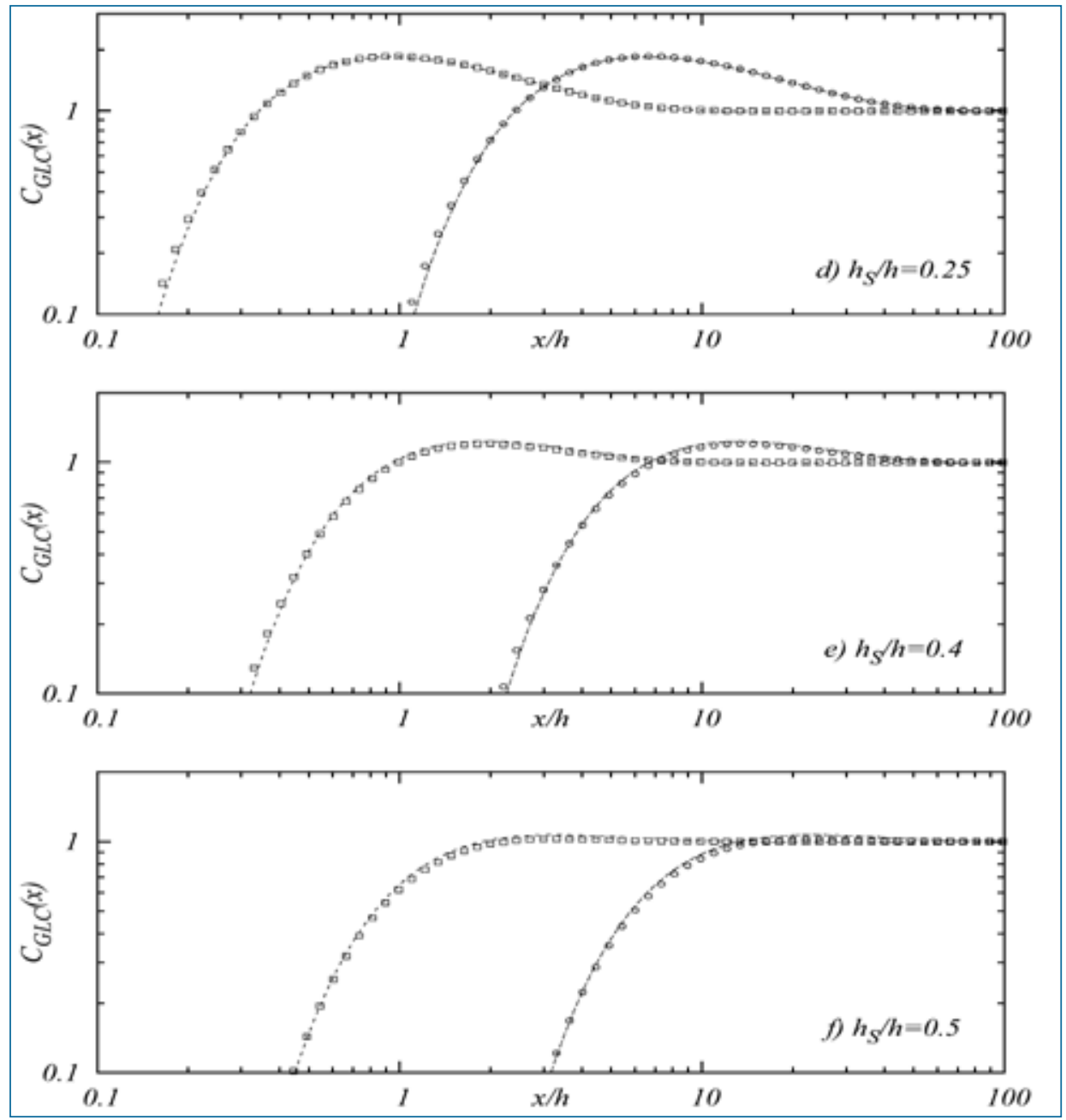

Figure $1 \mathrm{~b}$ - The GLC is plot versus $\stackrel{q}{\not /}$ for several source heights.

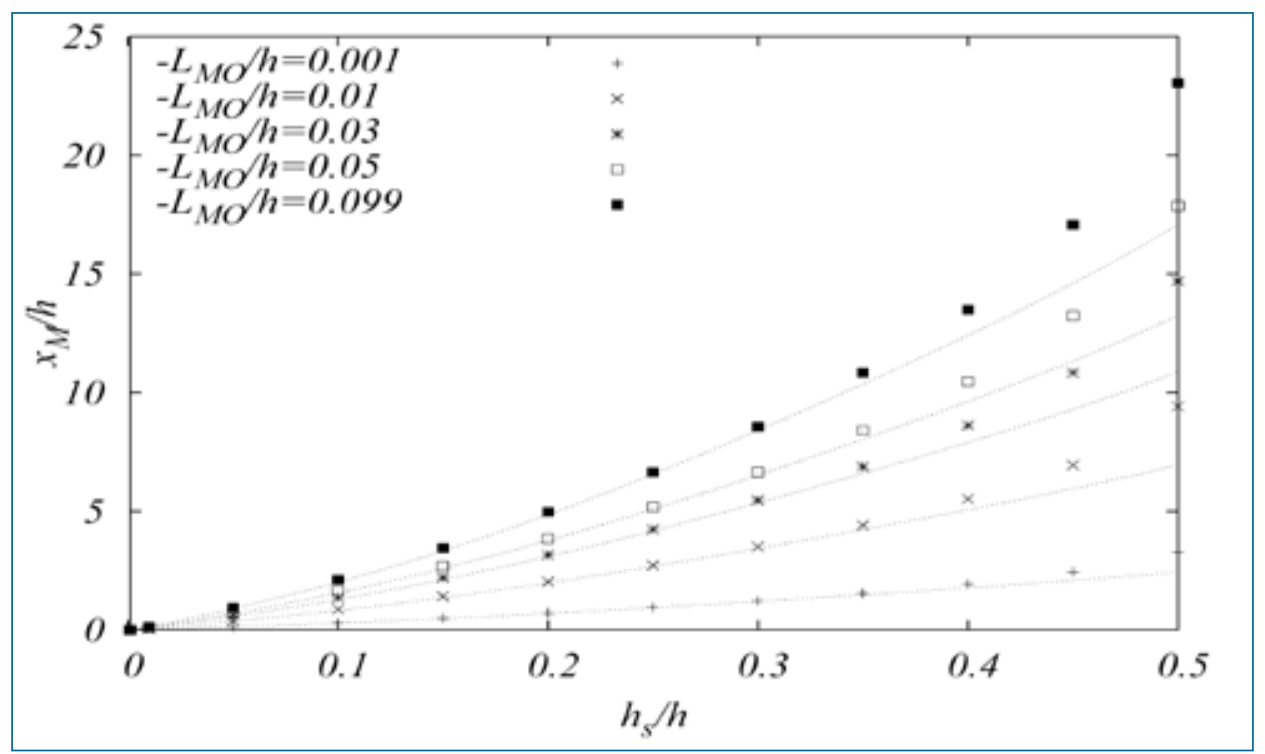

Figure 2 - Plot of $x_{M}$ versus $h_{S}^{\%}$. Points refer to the GILTT results, dotted lines refer to Eq. (16). 


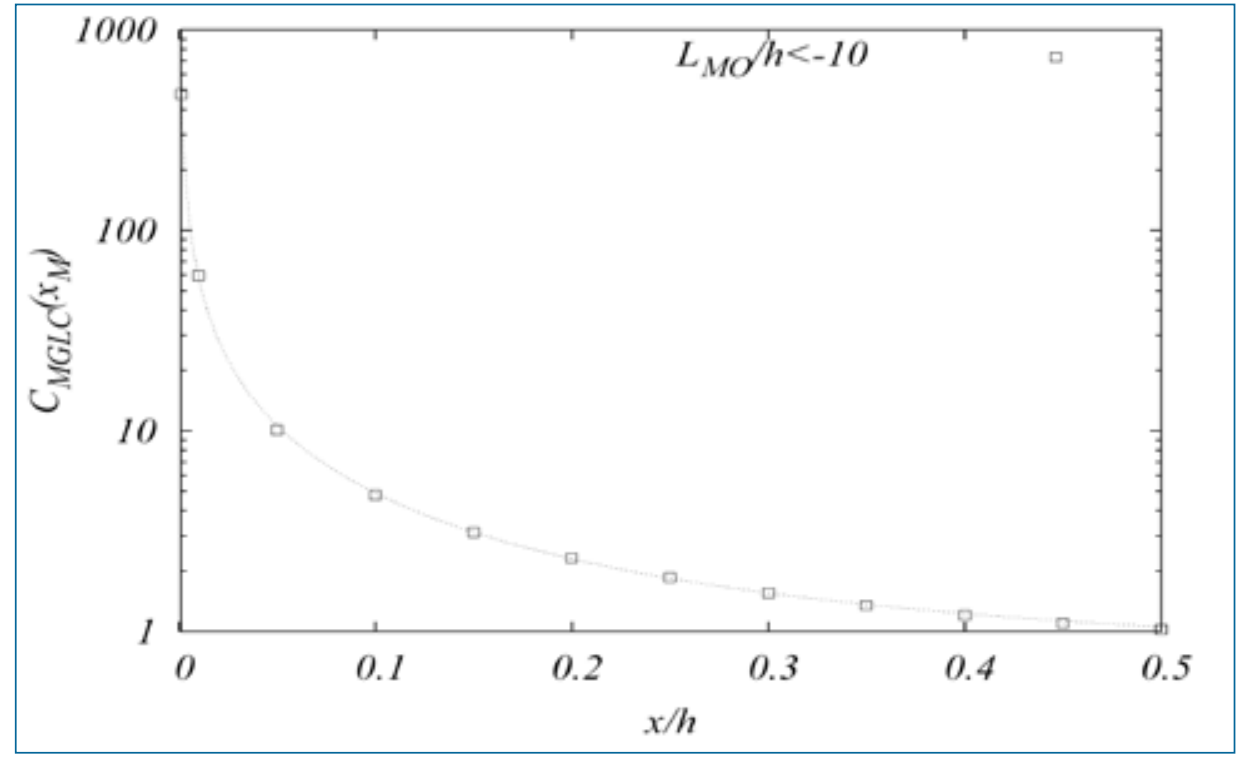

Figure 3 - Plot of $C_{M G L C}$ versus $h_{S}^{\circ}$. Points refer to the GILTT results, dotted lines refer to Eq. (17).

netheless, through the whole range of source heights $0<\widetilde{h}_{S} \leq 0.5$ the function $C_{G L C}(x)$ reproduces fairly well the GILTT results.

Figs. 2 and 3 show the position and value of maximum GLC respectively. These are scanned through the source height $h_{S}$ and for several selected values of the turbulence parameters $\widetilde{L}_{a}$. In both figures the GILTT results (points) are superimposed on the explicit approximations. Figure 2 depicts the position where the maximum occurs, for low sources dotted GILTT results and approximated lines (Eq. (13)) show good matching regardless the turbulence regime. For higher sources a mismatch occurs and the discrepancy increases as convective turbulence reduces strength, this fact follows from the condition (20).

Turbulence dependency shows that for a fixed $h_{S}$ the strength of convection causes therefor $x_{M}$ to get closer to the source height. From the physics point of view this result agrees with the mixing effect of turbulence. A final remark sh(did be made about Fig. 3. Both GILTT than expression (10) confirm that the maximum GLC value depends on the source height, regardless the turbulence. Based on the expression (10) and parameters definitions (11)-(14), respectively for b, c and k, the leading term for the maximum GLC results:

$$
C_{M G L C}\left(x_{M}\right) \approx h_{S}^{0}
$$

and the exponent -1 is a lower bound. These results broaden the well-known result obtained with the Gaussian approach for an unbounded ABL.

\section{Conclusions}

The GLC from an emitting point source in a steady convective ABL was expressed by a compact analytical expression.

The principal progresses worth emphasizing is that for a function given in Eq. (10), within the setting choice for the ABL parameter set, the maximum GLC depends only on the source height, regardless the Monin-Obukhov length. On the other hand, turbulence can still affect the position where the maximum GLC occurs, which is also confirmed by the GILTT solution.

On the operative point of view, the expression (10) and its related features are useful as an additional tool for decisional as well as emergency responses.

\section{References}

Buske D, Vilhena MT, Moreira DM, TirabassI T. Simulation of pollutant dispersion for low wind conditions in stable and convective planetary boundary layer. Atmospheric Environment. 2007; 41 (26): 5496-5501. 
Irwin JS. A theoretical variation of the wind profile power-low exponent as a function of surface roughness and stability. Atmospheric Environment. 1979; 13: 191-194.

Moreira DM, Vilhena MT, Buske D, Tirabassi T. The state-of-art of the GILTT method to simulate pollutant dispersion in the atmosphere. Atmospheric Research. 2009; 92 (1): 1-17.

Panofsky HA, Dutton JA. Atmospheric Turbulence. New York: John Wiley \& Sons; 1988.

Pasquill F, Smith FB. Atmospheric Diffusion. New York: John Wiley \& Sons; 1984.

Tiziano Tirabassi

Institute of Atmospheric Science and Climate (ISAC-CNR), Bologna, Itália E-mail: t.tirabassi@isac.cnr.it

\section{Daniela Buske}

Universidade Federal de Pelotas, Pelotas, RS, Brasil E-mail: danielabuske@gmail.com 